IJMS 17 (Special Issue), 119-141 (2010)

\title{
CRISES AND THE VOLATILITY OF INDONESIAN MACRO-INDICATORS ${ }^{1}$
}

\author{
CATUR SUGIYANTO \\ Faculty of Economics and Business \\ Universitas Gadjah Mada, Indonesia
}

\begin{abstract}
This paper examines the volatility of some of Indonesian macroeconomic indicators, namely the Bank Indonesia rate, inflation, and exchange rates. It is argued that after the financial crisis the variability of these variables increases and this makes it more difficult to predict them. The estimated $A R C H$ parameters increases overtime, indicating higher contribution of shock over several periods. From the random walk, historical mean, moving average and simple regression, it was found that the quality of prediction after the crisis decreases. Financial manager and other policy makers may adjust their strategy to account for this increase in variability.
\end{abstract}

\section{Introduction}

Financial time series, such as stock prices, exchange rates, and inflation rates often exhibit the phenomenon of volatility clustering, that is, periods in which their prices show wide swings for an extended time period followed by periods in which there is relative calm. Knowledge of volatility is of crucial importance in many areas. For example, financial planners may benefit from understanding the volatility of inflation (prices) in exercising financial plans, whereas importers, exporters, and traders in foreign exchange markets may be affected by the variability in the exchange rates as that might mean huge losses or profits. Likewise, for investors in the stock market, for high volatility in stock prices could mean huge losses or gains, and hence, greater uncertainty.

A series of financial crises have hit the Indonesian economy and the world for the last two decades. With the 2008 financial crises being the latest, the world has experienced crises in response to increase 
in oil prices (1974, 1978, 1984, and 2007-2008), and financial crises in 1998 and 2008. Such increase in oil prices raises the question if the macro-economic indicators have been more volatile recently. The main purpose of this research was to test whether there is evidence of increase in volatility of the Indonesian macro-economic indicators. Increased volatility means increased difficulty in predicting the indicators that may raise the risk and uncertainty to speculator. As a consequence, policies to influence the macro-indicators such as inflation targeting, may not be effective or may be difficult to achieve. So, accurate information on the macro-economic behaviour can be beneficial to both fund managers and policy makers.

This paper is outlined as follows. The next section provides the theoretical background for the study followed by the estimation strategy. Section four describes the recent development of macroeconomic indicators. Section five reports the estimation results and the last section concludes.

\section{Literature Review}

Volatility is the variability of the asset price changes over a particular period of time and it is sometimes difficult to predict correctly and consistently. Financial market volatility presents a strange paradox to the market participants, academicians, and policy makers - without volatility superior returns cannot be earned, since a risk free security offers meager returns. On the other hand, if it is high, it may lead to losses for the market participants and represent costs to the overall economy. However, there is question as to what model should be used to calculate volatility? The answer is not clear as different volatility models were proposed in the literature and were being used by practitioners and these varying models lead to different volatility estimates. In the past two decades this has been a fertile area for research in financial economics for both academicians as well as practitioners. Unfortunately most of the work was done in the context of developed markets in the context of stock and foreign exchange markets.

Poon and Granger (2003) provided an extensive review of the literature related to forecasting volatility. They divided the existing research into two general categories: (1) papers using historical data only and (2) papers using index volatility (IV) alone or in addition to historical data. In general, the latter studies had found that IV contains a significant amount of information and that it is often superior to models that rely on historical information alone. Since it is reasonable 
to assume that different markets have differing degrees of efficiency, the forecasting power of IV for one asset class does not necessarily mean that the IV will have equivalent capabilities in another. While the testing methodologies may be similar, the results of the IV tests should be considered according to asset class. As a consequence, it is argued that using the historical data may still be reasonable to forecast volatility.

A characteristic most of financial time series is that in their level form, they are random walks; that is, they are non-stationary. On the other hand, in the first difference form, they are generally stationary. Therefore, instead of modeling the levels of financial time series, its first difference is often considered, but these first differences often exhibit wide swings, or volatility, suggesting that the variance of financial time series varies over time. A model that fits with this behaviour is the Autoregressive Conditional Heteroscedasticity (ARCH) by Engle (1982).

Let $x$ be the variable that is considered, then $x^{2}$ is used as a measure of volatility (close to the variances or conditional variances). Being a squared quantity, its value will be high in periods when there are big changes, for example in the prices of financial assets and its value will be comparatively small when there are modest changes in the prices of financial assets. As $x^{2}$ measures volatility, the following $\operatorname{AR}(1)$, or ARIMA $(1,0,0)$, model is considered as:

$$
x_{t}^{2}=\beta_{0}+\beta_{1} x_{t-1}^{2}+u_{t}
$$

This model postulates that volatility in the current period is related to its value in the previous period plus a white noise error term. If $\beta_{1}$ is positive, it suggests that if volatility was high in the previous period, it will continue to be high in the current period, indicating volatility clustering. If $\beta_{1}$ is zero, then there is no volatility clustering.

A more complex model after the ARCH is GARCH (Generalised Autoregressive Conditional Heteroscedasticity) by Bollerslev (1986). The simplest form of GARCH is that the conditional variance of error at time $t$ depends not only on the squared error term in the previous time period (as in $\mathrm{ARCH}(1)$ ) but also on its conditional variance in the previous time period. The conditional variance of error at time $t$ depends not only on the squared error term in the previous time $(t-i$, as in $\mathrm{ARCH}(1))$ but also on its conditional variance in the previous time $(t-i)$. 
$u_{t}^{2}=\alpha_{0}+\alpha_{1} u_{t-1}^{2}+\alpha_{2} x_{t-1}^{2}$

It is then identified which one is stronger between $\alpha_{1}$ (the $\mathrm{ARCH}$ coefficient) and $\alpha_{2}$ (the GARCH coefficient). The ARCH coefficient measures the reaction of the conditional variance to shocks while the GARCH coefficient measures persistence. The stronger the $\mathrm{ARCH}$ coefficient then indicates that the larger shocks influence the conditional variances, which implies difficulties in predicting the variance.

\section{Estimation Strategy}

In this research, the evaluation of the volatility of the Indonesian macro economic indicators by using the GARCH model is proposed to and assess the difficulty in predicting the indicators by using: the random walk model, the moving average, and the simple regression.

\section{Random Walk Model}

As per this model, the best forecast for this period's volatility is the last period's realised:

$$
x_{t}^{2}=x_{t-1}^{2}
$$

\section{Moving Average Model}

- In the historic mean model, the forecast is based on all the available observations and each observation, whether it is very old or immediate, is given equal weight, and this may lead to stale prices affecting the forecasts. This is adjusted in a moving average method, which is a traditional time series technique in which the volatility is defined as the equally weighted average of realised volatilities in the past $i$ months. The choice of $i$ is rather arbitrary and in this paper, only the three month average was investigated.

$$
x_{t}^{2}=\frac{1}{3} \sum_{i=1}^{3} x_{t-i}^{2}
$$

\section{Simple Regression}

In this method, the familiar regression of actual volatilities on lagged values is run. In other words, it is the first autoregression performed 
on the first part of data which is meant for estimating the parameters, and the estimates thus obtained were used for forecasting the volatility for the next month. Accordingly the first part involves running the following regression:

$$
x_{t}^{2}=\alpha+\beta x_{t-1}^{2}
$$

$\alpha$ and $\beta$ are estimated over the periods of observation. It is assumed that the agent revises its parameters within these periods, i.e. the time varying parameters are only applied in three periods.

\section{Forecast Evaluation}

A qualitative forecast evaluation was used in this study. Following Naik and Leuthold (1986), a comparison of the actual and predicted turning point was performed. A $4 \times 4$ matrix was modified to document the change in variability, whether they have a smile, inverted smile, straight increase or decrease in every three months observation, and other changes were also added: constant-increase, increase-constant, constant-decrease, decrease-constant, and constant-constant. The shaded cells in the matrix (Table 1) represent the correct predictions and this paper will report the percentage errors in prediction.

\section{A Bird's-eye View on Indonesian Macroeconomic Indicators}

The period of observation was divided into three periods to indicate the change in the fluctuation in response to international changes. The following is a description of the development of the data.

\section{Bank Indonesia Rate}

The Bank Indonesia rate (BI rate) is the Bank Indonesia Certificate (Sertifikat Bank Indonesia) offered to the banks that will deposit their money with Bank Indonesia. There is a clear fluctuation, especially after the crisis in 1998. The root mean squares error increased from 1.24 (for period before the crisis 1998) to 2.65 (for period 1990-2008). The highest fluctuation occurred in 1998 when the domestic interest rate jumped up to $75 \%$ per year. The impact of the world financial crisis from the US was not dominant, since it was observed that the interest rate only increased slightly. In fact the recent rate was down, around $7 \%$. 


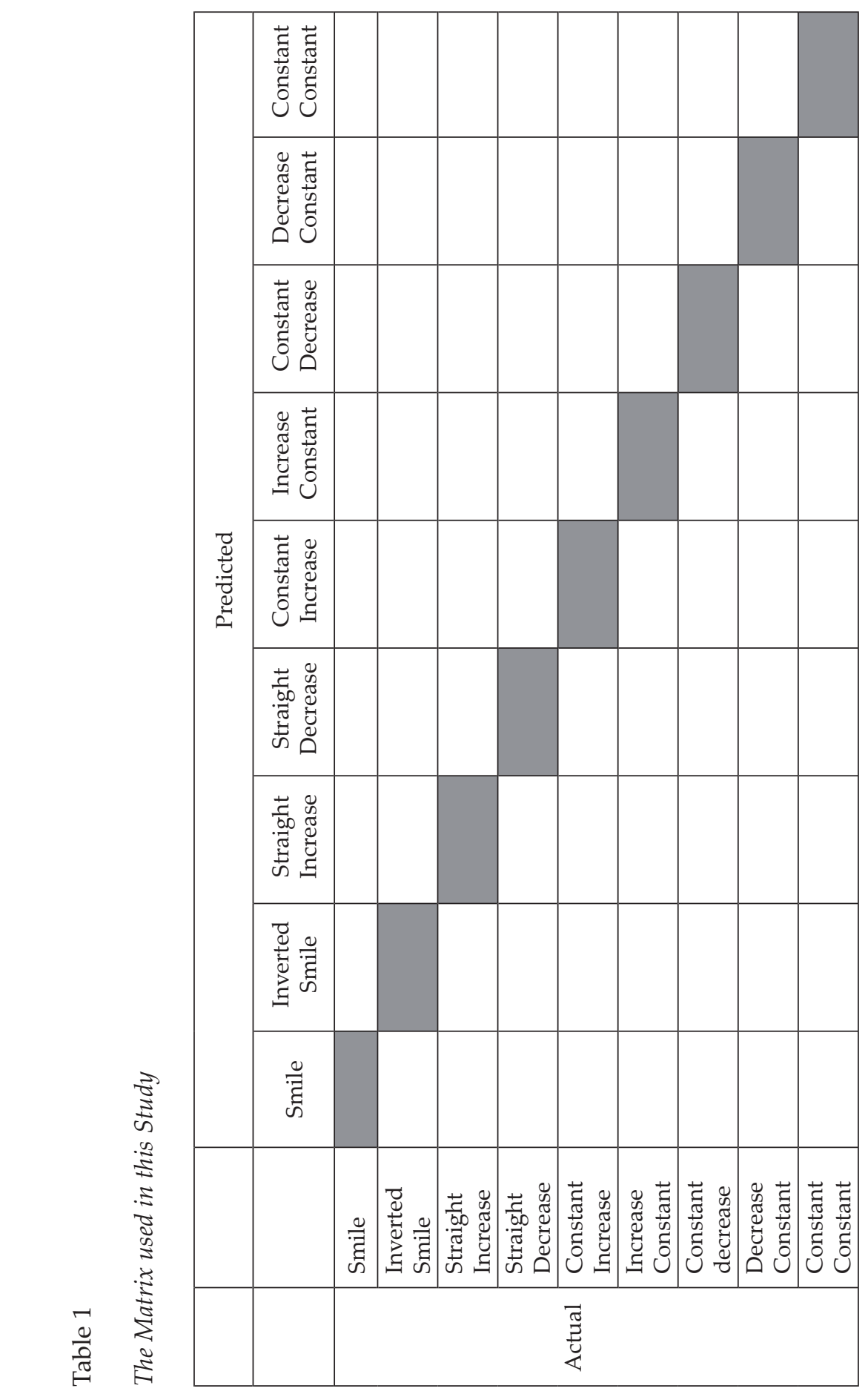

124 IJMS 17 (Special Issue), 119-141 (2010) 


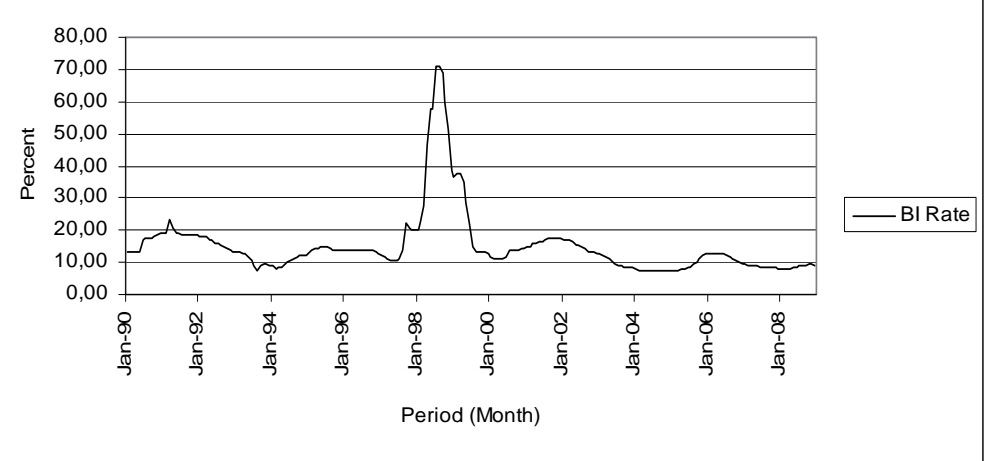

Figure 1. The Bank Indonesia (BI) Rate, January 1990-December 2008.

\section{Indonesian Rupiah Exchange Range}

A sharp change observed for the Indonesian Rupiah exchange rates. After the 1998 crisis, the rate fluctuated wildly. As the following figures show, the exchange rate was stable around Rp 2000 per US dollar before the 1998, then jumped to Rp 10000 in January 1998. After that the rate was moving at around this level. The root means squared error also jumped from 124.5 to 969.5 , confirming the above fluctuation.

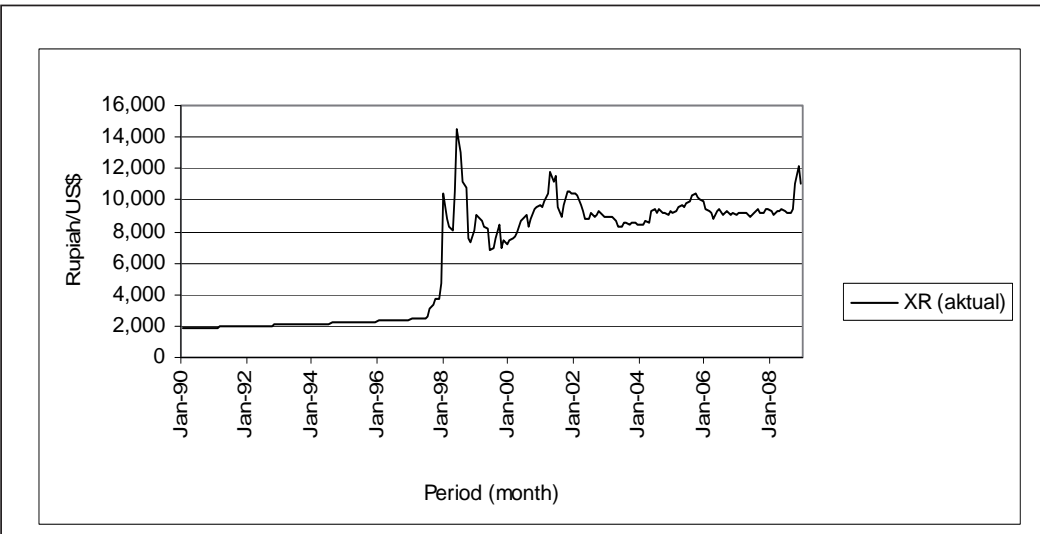

Figure 2. The Indonesian Rupiah Exchange Rates, XR (Rupiah/ US\$). 


\section{Inflation Rates}

The behaviour of the inflation was similar. Before the 1998 crisis, the inflation rates varied around $8 \%$, while after the crises it, was around $10 \%$. The range of fluctuation seems wider after the crises representing its higher variability. The big jump occurred during the 1998 crises.

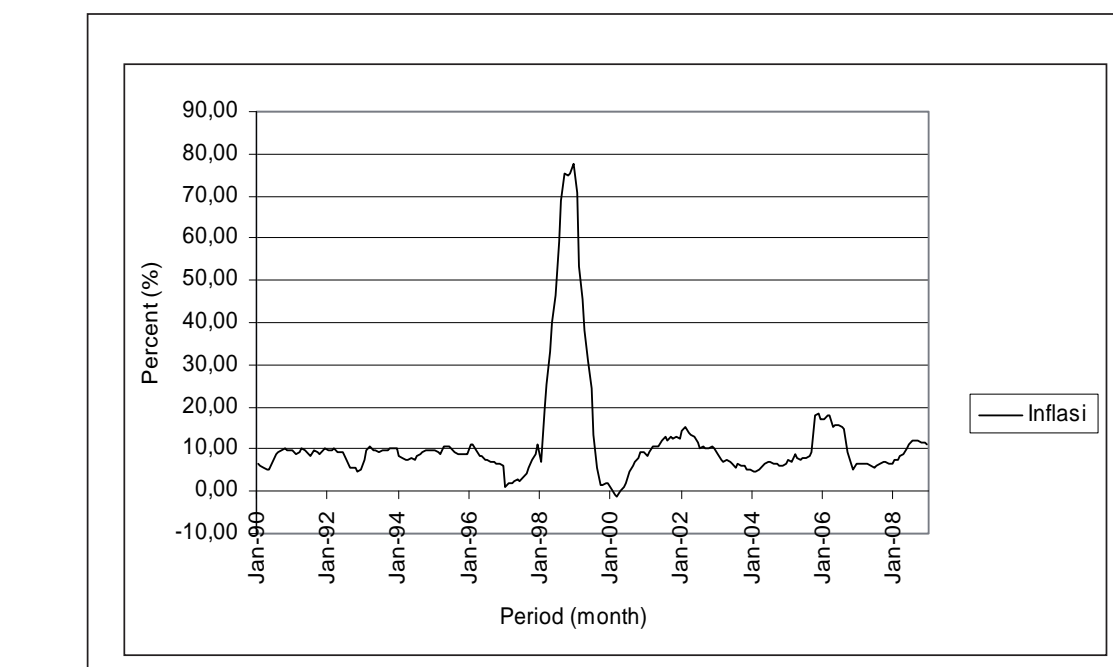

Figure 3. The Inflation Rates (Inflasi).

\section{Estimation Results}

In general there are two methods used in this study: (1) the predictability of the macro-indicators by using the random walk, historical mean, moving average, and simple regression, and (2) the estimation using GARCH. The predictability analysis was based on the matrix comparing the actual and the predicted, while the GARCH was used to indicate the ease of estimating the variables.

As mentioned, it is argued that when the volatility increases, the quality of prediction decreases. Table 1 reports the percentage errors in prediction. In general, the quality of prediction was not good. The values of percentage error of prediction were more than $50 \%$. This may indicate the quality of the method used in general. Other than that situation, inclusion of the 1998 crises period causes the prediction error of all periods 1990-2005 to become higher than those of 19901997. 
The BI rate prediction error increased from $62.5 \%$ to $86.4 \%$ (using the random walk). Surprisingly, the prediction error was zero when the moving average estimates were used. The exchange rates have been difficult to predict as well. The error in prediction increased from $43 \%$ to $60 \%$ (using the random walk model), $40 \%$ to $58 \%$, but decreased from $90 \%$ to $85 \%$ (using moving average).

When the period of observation was extended (1990-2008) the percentage error of prediction were not worse compared to those of period 1990-1997. It is argued that the longer the period, the better the quality of prediction. The complete report on prediction quality are presented in Appendix 1.

Table 1

Percentage Error of Prediction: Random Walk, Moving Average, and Simple OLS

\begin{tabular}{llccc}
\hline & & 1990-1997 & 1990-2005 & 1990-2008 \\
\hline \multirow{7}{*}{ BIRATE } & Random Walk & 62,5 & 86,4 & 62,5 \\
& Moving Average & 0,0 & 0,0 & 0,0 \\
& Simple OLS & 56,3 & 56,3 & 57,6 \\
\multirow{5}{*}{ Xrates } & Random Walk & 43,8 & 56,3 & 60,5 \\
& Moving Average & 96,9 & 85,1 & 85,7 \\
& Simple OLS & 40,6 & 53,1 & 58,7 \\
& & & & \\
& Randlation & 62,5 & 48,4 & 60,5 \\
& Moving Average & 0,0 & 0,0 & 0,0 \\
& Simple OLS & 53,3 & 63,8 & 60,0 \\
\hline
\end{tabular}

The above analysis did not provide a firm conclusion, whether the variables had become difficult to predict after the crisis. The following are the ARCH and GARCH estimates to account for further variability. As Table 2 reports, in all of the estimations it was observed that almost all of the ARCH and GARCH coefficients are significant. These indicated that both the shock and the persistence (the past variance) determine the current conditional variance. All of the GARCH coefficients are higher than those of the $\mathrm{ARCH}$, indicating that the persistence of variances are higher compared to the temporary shocks. In terms of variability, the comparison of the three periods showed that the ARCH coefficients are more significant, confirming the increase significance of the shock. The ARCH coefficients increase 
steadily over the three periods, supporting the importance of shock in forming the variance. The forecast and adjusted samples also confirm the above estimated $\mathrm{ARCH}$ and GARCH. The figure are reported in Appendix 2.

\section{Table 2}

\section{ARCH and GARCH Coefficient Estimates and their $z$ Statistics}

\begin{tabular}{|c|c|c|c|c|c|c|}
\hline & \multicolumn{2}{|c|}{ 1990-1997 } & \multicolumn{2}{|c|}{ 1990-2005 } & \multicolumn{2}{|c|}{ 1990-2008 } \\
\hline & $\alpha_{1}$ & $\alpha_{2}$ & $\alpha_{1}$ & $\alpha_{2}$ & $\alpha_{1}$ & $\alpha_{2}$ \\
\hline BI Rate & $\begin{array}{c}0.425 \\
(5.86)^{* * *}\end{array}$ & $\begin{array}{c}-0.04 \\
(-14.9)^{* * *}\end{array}$ & $\begin{array}{c}2.17 \\
(6.05)^{* * *}\end{array}$ & $\begin{array}{l}0.21 \\
(8.27)^{* * *}\end{array}$ & $\begin{array}{l}2.09 \\
(7.37)^{* * *}\end{array}$ & $\begin{array}{c}0.22 \\
(9.43)^{* * *}\end{array}$ \\
\hline Exchange Rate & $\begin{array}{c}0.39 \\
(10.0)^{* * *}\end{array}$ & $\begin{array}{c}0.94 \\
(120.0)^{* * *}\end{array}$ & $\begin{array}{l}1.65 \\
(9.32)^{* * *}\end{array}$ & $\begin{array}{c}0.51 \\
(12.7)^{* * *}\end{array}$ & $\begin{array}{c}2.12 \\
(12.3)^{* * * *}\end{array}$ & $\begin{array}{c}0.44 \\
(13.5)^{* * *}\end{array}$ \\
\hline Inflation Rate & $\begin{array}{c}-0.01 \\
(-1.05)\end{array}$ & $\begin{array}{r}1.03 \\
(514)^{* * *}\end{array}$ & $\begin{array}{c}0.79 \\
(5.61)^{* * *}\end{array}$ & $\begin{array}{c}-0.04 \\
(224)^{* * *}\end{array}$ & $\begin{array}{l}0.73 \\
(6.04)^{* * *}\end{array}$ & $\begin{array}{l}-0.04 \\
(-365)^{* * *}\end{array}$ \\
\hline
\end{tabular}

Notes. Figures in parentheses are $\mathrm{z}$ statistics: significant at $0.1,{ }^{* *} 0.5$, and $* * *$ 0.01 .

\section{Conclusion}

This research tested whether many Indonesian macroeconomic indicators have become more volatile after the financial crises of 1998 and 2008. In order to examine that behaviour, the Bank Indonesia rates, inflation, and exchange rates were used. Three methods of estimation were implemented, namely random walk, moving average, and simple OLS, which employed ARCH and GARCH estimates. The observation was also divided into three periods: 1990-1997, 19902005, and 1990-2008, to account for the changes in these periods.

It was concluded that the volatility of the selected macroeconomic indicators increased after the 1998 crises. The shock component (the $\mathrm{ARCH}$ parameter) increased after the crises, both in terms of the size of the parameters and their significant levels. A peculiar change was observed in the exchange rates, which also rised from Rp 3000 per US dollar to around Rp 10000 per US dollar.

Policy makers should be aware that the Indonesian macroeconomic indicators have increased their volatility. Traders and fund managers 
should consider such increase in variability as an opportunity to define an accurate trading decision to gain from the fluctuation.

This study did not obtain estimators that show a consistent increase of the prediction error after the crises. Other estimators may be explored in future studies to measure the variability of the indicators.

\section{Acknowledgements}

1. We would like to thank the anonymous referee for the comments. We also would like to thank Daniel Silalahi for his excellent research assistant. As usual, I am responsible for any errors and omissions.

\section{Endnotes}

1. In this research, the indicators are: exchange rates, interest rate (BI rate), and inflation (CPI).

\section{References}

Bollerslev. (1986). Generalized autoregressive conditional heteroscedasticity. Journal of Econometrics, 31, 307-326.

Gudjarati, D. (2004). Basic econometrics (4th ed.). New York: Mc Graw-Hill.

Naik, G., \& R.M. Leuthold. (1986). A note on qualitative forecast evaluation. American Journal of Agricultural Economics, 68, 721726

Poon, S., \& C.W.J. Granger. (2003). Forecasting volatility in financial markets: A review. Journal of Economic Literature, 478-539.

Engle. R. (1982). Autoregressive conditional heteroscedasticity with estimates of the variance of United Kingdom inflation. Econometrica, 50(1), 987-1007. 
Appendix 1. Qualitative Prediction Evalution

AKTUAL BIR (1990-1997), Random Walk

\begin{tabular}{|c|c|c|c|c|c|c|c|c|c|c|}
\hline \multirow[b]{5}{*}{ PREDIKSI } & & $\begin{array}{l}\text { naik- } \\
\text { turun }\end{array}$ & $\begin{array}{c}\text { turun- } \\
\text { naik }\end{array}$ & $\begin{array}{c}\text { naik- } \\
\text { naik }\end{array}$ & $\begin{array}{l}\text { turun- } \\
\text { turun }\end{array}$ & $\begin{array}{l}\text { naik- } \\
\text { tetap }\end{array}$ & $\begin{array}{c}\text { tetap- } \\
\text { naik }\end{array}$ & $\begin{array}{l}\text { turun- } \\
\text { tetap }\end{array}$ & $\begin{array}{l}\text { tetap- } \\
\text { turun }\end{array}$ & $\begin{array}{l}\text { tetap- } \\
\text { tetap }\end{array}$ \\
\hline & naik-turun & & 2 & & 1 & & & & & \\
\hline & turun-naik & & & & & 3 & & & & \\
\hline & naik-naik & & & 6 & & 1 & & & & \\
\hline & turun-turun & & 1 & & 6 & & & 2 & & 1 \\
\hline & naik-tetap & & & 1 & & & 1 & & & \\
\hline & tetap-naik & & & & & & & & & \\
\hline & turun-tetap & & & & & & & & 2 & \\
\hline & tetap-turun & & 1 & & 3 & & & & & \\
\hline & tetap-tetap & & & & & & & & 1 & \\
\hline
\end{tabular}

AKTUAL XR (1990-2005)

\begin{tabular}{|l|c|c|c|c|c|c|c|c|c|}
\hline & $\begin{array}{l}\text { naik- } \\
\text { turun }\end{array}$ & $\begin{array}{c}\text { Turun } \\
\text {-naik }\end{array}$ & $\begin{array}{c}\text { naik- } \\
\text { naik }\end{array}$ & $\begin{array}{c}\text { Turun } \\
\text {-turun }\end{array}$ & $\begin{array}{c}\text { naik- } \\
\text { tetap }\end{array}$ & $\begin{array}{c}\text { tetap- } \\
\text { naik }\end{array}$ & $\begin{array}{c}\text { turun- } \\
\text { tetap }\end{array}$ & $\begin{array}{c}\text { tetap- } \\
\text { turun }\end{array}$ & $\begin{array}{c}\text { tetap- } \\
\text { tetap }\end{array}$ \\
\hline naik-turun & & 2 & & 1 & & & & & \\
\hline turun-naik & & & 3 & 1 & 3 & & & & \\
\hline naik-naik & 1 & & 6 & 1 & 1 & & & & \\
\hline turun-turun & & 1 & 1 & 11 & & & 2 & & 1 \\
\hline naik-tetap & & & & & & 1 & & & \\
\hline tetap-naik & & & 3 & & & & & & \\
\hline turun-tetap & & & & & & & & 2 & \\
\hline tetap-turun & & & & 1 & & & & & \\
\hline tetap-tetap & & & & & & 1 & & 1 & \\
\hline
\end{tabular}

AKTUAL XR (1990-2008)

\begin{tabular}{|c|c|c|c|c|c|c|c|c|c|c|}
\hline \multirow{10}{*}{ PREDIKSI } & & $\begin{array}{l}\text { naik- } \\
\text { turun }\end{array}$ & $\begin{array}{l}\text { turun- } \\
\text { naik }\end{array}$ & $\begin{array}{l}\text { naik- } \\
\text { naik }\end{array}$ & $\begin{array}{l}\text { Turun } \\
\text {-turun }\end{array}$ & $\begin{array}{l}\text { naik- } \\
\text { tetap }\end{array}$ & $\begin{array}{l}\text { tetap- } \\
\text { naik }\end{array}$ & $\begin{array}{c}\text { turun- } \\
\text { tetap }\end{array}$ & $\begin{array}{l}\text { tetap- } \\
\text { turun }\end{array}$ & $\begin{array}{l}\text { tetap- } \\
\text { tetap }\end{array}$ \\
\hline & naik-turun & & 2 & & 1 & 1 & & & & \\
\hline & turun-naik & & & 3 & 1 & 3 & & & & \\
\hline & naik-naik & 1 & & 7 & 1 & 1 & & & 1 & \\
\hline & turun-turun & & 1 & 1 & 14 & & & 2 & & 1 \\
\hline & naik-tetap & & & & & & 1 & & & \\
\hline & tetap-naik & & & 4 & & & & & & \\
\hline & turun-tetap & & & & & & & & 2 & 1 \\
\hline & tetap-turun & & & & 3 & & & & & \\
\hline & tetap-tetap & & & & & & 1 & & 2 & 1 \\
\hline
\end{tabular}




\begin{tabular}{|c|c|c|c|c|c|c|c|c|c|c|}
\hline \multicolumn{3}{|c|}{ Moving Average } & \multicolumn{5}{|c|}{ AKTUAL BIR (1990-1997) } & \multirow[b]{2}{*}{$\begin{array}{l}\text { turun- } \\
\text { tetap }\end{array}$} & \multirow[b]{2}{*}{$\begin{array}{l}\text { tetap- } \\
\text { turun }\end{array}$} & \multirow[b]{2}{*}{$\begin{array}{l}\text { tetap } \\
\text { tetap }\end{array}$} \\
\hline \multirow{10}{*}{ PREDIKSI } & & $\begin{array}{l}\text { naik- } \\
\text { turun }\end{array}$ & $\begin{array}{l}\text { Turun } \\
\text {-naik }\end{array}$ & $\begin{array}{c}\text { naik- } \\
\text { naik }\end{array}$ & $\begin{array}{l}\text { turun- } \\
\text { turun }\end{array}$ & $\begin{array}{l}\text { naik- } \\
\text { tetap }\end{array}$ & $\begin{array}{l}\text { tetap } \\
\text { naik }\end{array}$ & & & \\
\hline & naik-turun & & & & & & & & & \\
\hline & turun-naik & & 4 & & & & & & & \\
\hline & naik-naik & & & 7 & & & & & & \\
\hline & turun-turun & & & & 10 & & & & & \\
\hline & naik-tetap & & & & & 4 & & & & \\
\hline & tetap-naik & & & & & & 1 & & & \\
\hline & turun-tetap & & & & & & & 3 & & \\
\hline & tetap-turun & & & & & & & & 3 & \\
\hline & tetap-tetap & & & & & & & & & \\
\hline
\end{tabular}

AKTUAL BIR (1990-2005)

\begin{tabular}{|c|c|c|c|c|c|c|c|c|c|c|c|}
\hline \multirow{10}{*}{ PREDIKSI } & & $\begin{array}{l}\text { naik- } \\
\text { turun }\end{array}$ & $\begin{array}{l}\text { turun- } \\
\text { naik }\end{array}$ & $\begin{array}{l}\text { naik- } \\
\text { naik }\end{array}$ & $\begin{array}{l}\text { turun- } \\
\text { turun }\end{array}$ & $\begin{array}{l}\text { naik- } \\
\text { tetap }\end{array}$ & $\begin{array}{c}\text { teta } \\
\text { nai }\end{array}$ & & $\begin{array}{l}\text { Turun } \\
\text {-tetap }\end{array}$ & $\begin{array}{l}\text { Tetap- } \\
\text { turun }\end{array}$ & $\begin{array}{l}\text { tetap- } \\
\text { tetap }\end{array}$ \\
\hline & naik-turun & 1 & & & & & & & & & \\
\hline & turun-naik & & 1 & & & & & & & & \\
\hline & naik-naik & & & 12 & & & & & & & \\
\hline & turun-turun & & & & 15 & & & & & & \\
\hline & naik-tetap & & & & & 1 & & & & & \\
\hline & tetap-naik & & & & & & 2 & & & & \\
\hline & turun-tetap & & & & & & & & 3 & & \\
\hline & tetap-turun & & & & & & & & & 3 & \\
\hline & tetap-tetap & & & & & & & & & & \\
\hline
\end{tabular}

AKTUAL BIR (1990-2008)

\begin{tabular}{|l|l|l|l|l|l|l|l|l|l|l|}
\hline & $\begin{array}{c}\text { naik- } \\
\text { turun }\end{array}$ & $\begin{array}{c}\text { turun- } \\
\text { naik }\end{array}$ & $\begin{array}{c}\text { naik- } \\
\text { naik }\end{array}$ & $\begin{array}{c}\text { turun- } \\
\text { turun }\end{array}$ & $\begin{array}{c}\text { Naik } \\
\text {-tetap }\end{array}$ & $\begin{array}{c}\text { tetap- } \\
\text { naik }\end{array}$ & $\begin{array}{c}\text { turun- } \\
\text { tetap }\end{array}$ & $\begin{array}{c}\text { tetap- } \\
\text { turun }\end{array}$ & $\begin{array}{c}\text { tetap- } \\
\text { tetap }\end{array}$ \\
\hline PREDIKSI & 1 & & & & & & & & \\
\hline turun-naik & & 1 & & & & & & & \\
\hline & naik-naik & & & 14 & & & & & & \\
\hline & turun-turun & & & & 20 & & & & & \\
\hline & naik-tetap & & & & & 1 & & & & \\
\hline tetap-naik & & & & & & 2 & & & \\
\hline turun-tetap & & & & & & & 4 & & \\
\hline tetap-turun & & & & & & & & 5 & \\
\hline tetap-tetap & & & & & & & & & 2 \\
\hline
\end{tabular}




\begin{tabular}{|c|c|c|c|c|c|c|c|c|c|}
\hline & $\begin{array}{l}\text { naik- } \\
\text { turun }\end{array}$ & $\begin{array}{c}\text { turun- } \\
\text { naik }\end{array}$ & $\begin{array}{l}\text { naik- } \\
\text { naik }\end{array}$ & \begin{tabular}{|l} 
Turun \\
-turun
\end{tabular} & $\begin{array}{l}\text { naik- } \\
\text { tetap }\end{array}$ & $\begin{array}{l}\text { tetap } \\
\text { naik }\end{array}$ & turur & $\begin{array}{l}\text { Tetap- } \\
\text { turun }\end{array}$ & $\begin{array}{l}\text { tetap- } \\
\text { tetap }\end{array}$ \\
\hline naik-turun & & 2 & & 1 & & & & & \\
\hline turun-naik & & 2 & & & & & & & \\
\hline naik-naik & & & 7 & & 1 & 1 & & & \\
\hline turun-turun & & 1 & & 5 & & & 3 & & \\
\hline \multicolumn{10}{|l|}{ naik-tetap } \\
\hline \multicolumn{10}{|l|}{ tetap-naik } \\
\hline turun-tetap & & & & & & & & 2 & \\
\hline tetap-turun & & 1 & & 4 & & & & & \\
\hline tetap-tetap & & & & & 1 & & & 1 & \\
\hline
\end{tabular}

AKTUAL BIR (1990-2005)

\begin{tabular}{|l|c|c|c|c|c|c|c|c|c|}
\hline & $\begin{array}{l}\text { naik- } \\
\text { turun }\end{array}$ & $\begin{array}{c}\text { turun- } \\
\text { naik }\end{array}$ & $\begin{array}{c}\text { naik- } \\
\text { naik }\end{array}$ & $\begin{array}{c}\text { Turun } \\
\text {-turun }\end{array}$ & $\begin{array}{c}\text { naik- } \\
\text { tetap }\end{array}$ & $\begin{array}{c}\text { tetap- } \\
\text { naik }\end{array}$ & $\begin{array}{c}\text { turun- } \\
\text { tetap }\end{array}$ & $\begin{array}{c}\text { Tetap } \\
\text {-turun }\end{array}$ & $\begin{array}{c}\text { tetap- } \\
\text { tetap }\end{array}$ \\
\hline naik-turun & & 2 & & 1 & & & & & \\
\hline turun-naik & & 2 & 4 & & & & & & \\
\hline naik-naik & 1 & & 7 & & 1 & 1 & & & \\
\hline turun-turun & & 1 & & 12 & & & 3 & & \\
\hline naik-tetap & & & & & & 1 & & & \\
\hline tetap-naik & & & 2 & & & & & & \\
\hline turun-tetap & & & & & & & & 2 & \\
\hline tetap-turun & & 1 & & 4 & & & & & \\
\hline tetap-tetap & & & & & 1 & 1 & & 1 & \\
\hline
\end{tabular}

AKTUAL BIR (1990-2008)

\begin{tabular}{|l|c|c|c|c|c|c|c|c|c|}
\hline & $\begin{array}{l}\text { naik- } \\
\text { turun }\end{array}$ & $\begin{array}{c}\text { turun- } \\
\text { naik }\end{array}$ & $\begin{array}{c}\text { naik- } \\
\text { naik }\end{array}$ & $\begin{array}{c}\text { turun } \\
\text {-turun }\end{array}$ & $\begin{array}{c}\text { naik- } \\
\text { tetap }\end{array}$ & $\begin{array}{c}\text { tetap- } \\
\text { naik }\end{array}$ & $\begin{array}{c}\text { turun- } \\
\text { tetap }\end{array}$ & $\begin{array}{c}\text { Tetap } \\
\text {-turun }\end{array}$ & $\begin{array}{c}\text { tetap- } \\
\text { tetap }\end{array}$ \\
\hline naik-turun & & 2 & & 1 & & & & & \\
\hline turun-naik & & 2 & 4 & & & & & & \\
\hline naik-naik & 1 & & 8 & & 1 & 1 & & & \\
\hline turun-turun & & 1 & & 15 & & & 3 & & \\
\hline naik-tetap & & & & & & 1 & & 1 & \\
\hline tetap-naik & & & 3 & & & & & & \\
\hline turun-tetap & & & & & & & & 2 & 1 \\
\hline tetap-turun & & 1 & & 6 & & & 1 & & \\
\hline tetap-tetap & & & & & 1 & 1 & & 1 & 1 \\
\hline
\end{tabular}


Random Walk

AKTUAL XR (1990-1997)

\begin{tabular}{|c|c|c|c|c|c|c|c|c|c|c|c|}
\hline & & $\begin{array}{l}\text { naik- } \\
\text { turun }\end{array}$ & $\begin{array}{c}\text { turun } \\
\text { naik }\end{array}$ & $\begin{array}{l}\text { naik- } \\
\text { naik }\end{array}$ & $\begin{array}{l}\text { turun- } \\
\text { turun }\end{array}$ & $\begin{array}{l}\text { naik } \\
\text { tetap }\end{array}$ & tetap & $\begin{array}{l}\text { turu } \\
\text { teta }\end{array}$ & & $\begin{array}{l}\text { tetap- } \\
\text { turun }\end{array}$ & $\begin{array}{l}\text { tetap- } \\
\text { tetap }\end{array}$ \\
\hline & naik-turun & & 1 & & & & & & & & \\
\hline & turun-naik & & & 3 & & & & & & & \\
\hline & naik-naik & 3 & 1 & 18 & & 1 & & & & & \\
\hline \multirow[t]{6}{*}{ PREDIKSI } & turun-turun & & 2 & & & & & 1 & & & \\
\hline & naik-tetap & & & & & & 2 & & & & \\
\hline & tetap-naik & & & & & & & & & & \\
\hline & turun-tetap & & & & & & & & & & \\
\hline & tetap-turun & & & & & & & & & & \\
\hline & tetap-tetap & & & & & & & & & & \\
\hline
\end{tabular}

AKTUAL BIR (1990-2005)

\begin{tabular}{|c|c|c|c|c|c|c|c|c|c|c|c|c|}
\hline & & $\begin{array}{l}\text { naik- } \\
\text { turun }\end{array}$ & $\begin{array}{r}\text { turun } \\
\text { naik }\end{array}$ & $\begin{array}{l}\text { naik } \\
\text { naik }\end{array}$ & $\begin{array}{l}\text { turun } \\
\text { turun }\end{array}$ & $\begin{array}{l}\text { naik } \\
\text { tetap }\end{array}$ & $\begin{array}{l}\text { tet } \\
\text { na }\end{array}$ & & $\begin{array}{l}\text { turu } \\
\text { teta }\end{array}$ & & $\begin{array}{l}\text { tetap- } \\
\text { turun }\end{array}$ & $\begin{array}{l}\text { tetap- } \\
\text { tetap }\end{array}$ \\
\hline & naik-turun & & 5 & 1 & 3 & & & & & & & \\
\hline & turun-naik & 2 & & 8 & & & & & & & & \\
\hline & naik-naik & 5 & 1 & 23 & & 1 & & & & & & \\
\hline \multirow[t]{6}{*}{ PREDIKSI } & turun-turun & & 6 & & 5 & & & & 1 & & & \\
\hline & naik-tetap & & & & 1 & & 2 & & & & & \\
\hline & tetap-naik & & & & & & & & & & & \\
\hline & turun-tetap & & & & & & & & & & & \\
\hline & tetap-turun & & & & & & & & & & & \\
\hline & tetap-tetap & & & & & & & & & & & \\
\hline
\end{tabular}

AKTUAL BIR (1990-2008)

\begin{tabular}{|c|c|c|c|c|c|c|c|c|c|c|}
\hline & & $\begin{array}{l}\text { naik- } \\
\text { turun }\end{array}$ & $\begin{array}{r}\text { turun } \\
\text { naik }\end{array}$ & $\begin{array}{l}\text { naik } \\
\text { naik }\end{array}$ & $\begin{array}{l}\text { turun- } \\
\text { turun }\end{array}$ & $\begin{array}{l}\text { naik- } \\
\text { tetap }\end{array}$ & $\begin{array}{c}\text { tetap } \\
\text { naik }\end{array}$ & $\begin{array}{l}\text { turun- } \\
\text { tetap }\end{array}$ & $\begin{array}{l}\text { tetap- } \\
\text { turun }\end{array}$ & $\begin{array}{l}\text { tetap- } \\
\text { tetap }\end{array}$ \\
\hline & naik-turun & & 5 & 1 & 3 & & & & & \\
\hline & turun-naik & 3 & & 11 & & & & & & \\
\hline & naik-naik & 9 & 1 & 24 & & 1 & & & & \\
\hline \multirow[t]{6}{*}{ PREDIKSI } & turun-turun & & 8 & & 6 & & & 1 & & \\
\hline & naik-tetap & . & & & 1 & & 2 & & & \\
\hline & tetap-naik & & & & & & & & & \\
\hline & turun-tetap & & & & & & & & & \\
\hline & tetap-turun & & & & & & & & & \\
\hline & tetap-tetap & & & & & & & & & \\
\hline
\end{tabular}


AKTUAL XR (1990-1997)

\begin{tabular}{|l|l|l|l|l|l|l|l|l|l|}
\hline & $\begin{array}{l}\text { naik- } \\
\text { turun }\end{array}$ & turun- & naik- & turun- \\
naik & naik- & tetap- & turun- \\
turun & tetap & naik & tetap & tetap- \\
turun & tetap \\
\hline naik-turun & & & 1 & & & & & & \\
\hline turun-naik & & & & & & & & & \\
\hline naik-naik & & & & & & & & & \\
\hline turun-turun & 3 & 3 & 20 & 1 & 1 & 2 & 1 & & \\
\hline naik-tetap & & & & & & & & & \\
\hline tetap-naik & & & & & & & & & \\
\hline turun-tetap & & & & & & & & & \\
\hline tetap-turun & & & & & & & & & \\
\hline tetap-tetap & & & & & & & & & \\
\hline
\end{tabular}

AKTUAL xr (1990-2005)

\begin{tabular}{|c|c|c|c|c|c|c|c|c|c|}
\hline & $\begin{array}{l}\text { naik- } \\
\text { turun }\end{array}$ & $\begin{array}{l}\text { turun- } \\
\text { naik }\end{array}$ & $\begin{array}{l}\text { naik- } \\
\text { naik }\end{array}$ & $\begin{array}{l}\text { turun- } \\
\text { turun }\end{array}$ & $\begin{array}{l}\text { naik- } \\
\text { tetap }\end{array}$ & $\begin{array}{c}\text { tetap } \\
\text { naik }\end{array}$ & $\begin{array}{l}\text { turun } \\
\text { tetap }\end{array}$ & tetap- & $\begin{array}{l}\text { tetap- } \\
\text { tetap }\end{array}$ \\
\hline naik-turun & & & 1 & & & & & & \\
\hline \multicolumn{10}{|l|}{ turun-naik } \\
\hline \multicolumn{10}{|l|}{ naik-naik } \\
\hline turun-turun & 7 & 14 & 31 & 10 & 1 & 2 & 1 & & \\
\hline \multicolumn{10}{|l|}{ naik-tetap } \\
\hline \multicolumn{10}{|l|}{ tetap-naik } \\
\hline \multicolumn{10}{|l|}{ turun-tetap } \\
\hline \multicolumn{10}{|l|}{ tetap-turun } \\
\hline tetap-tetap & & & & & & & & & \\
\hline
\end{tabular}

AKTUAL xr (1990-2008)

\begin{tabular}{|l|c|c|c|c|c|c|c|c|c|}
\hline & $\begin{array}{c}\text { naik- } \\
\text { turun }\end{array}$ & $\begin{array}{c}\text { turun- } \\
\text { naik }\end{array}$ & $\begin{array}{c}\text { naik- } \\
\text { naik }\end{array}$ & $\begin{array}{c}\text { turun- } \\
\text { turun }\end{array}$ & $\begin{array}{c}\text { naik- } \\
\text { tetap }\end{array}$ & $\begin{array}{c}\text { tetap- } \\
\text { naik }\end{array}$ & $\begin{array}{c}\text { turun- } \\
\text { tetap }\end{array}$ & $\begin{array}{l}\text { tetap- } \\
\text { turun }\end{array}$ & $\begin{array}{l}\text { tetap- } \\
\text { tetap }\end{array}$ \\
\hline naik-turun & & & 1 & & & & & & \\
\hline turun-naik & & & & & & & & & \\
\hline naik-naik & & & & & & & & & \\
\hline turun-turun & 10 & 16 & 35 & 11 & 1 & 2 & 1 & & \\
\hline naik-tetap & & & & & & & & & \\
\hline tetap-naik & & & & & & & & & \\
\hline turun-tetap & & & & & & & & & \\
\hline tetap-turun & & & & & & & & & \\
\hline tetap-tetap & & & & & & & & & \\
\hline
\end{tabular}


Simple OLS

\begin{tabular}{|c|c|c|c|c|c|c|c|c|c|c|}
\hline & & $\begin{array}{l}\text { naik- } \\
\text { turun }\end{array}$ & $\begin{array}{c}\text { turun- } \\
\text { naik }\end{array}$ & $\begin{array}{c}\text { naik- } \\
\text { naik }\end{array}$ & $\begin{array}{l}\text { turun- } \\
\text { turun }\end{array}$ & $\begin{array}{l}\text { naik- } \\
\text { tetap }\end{array}$ & $\begin{array}{l}\text { tetap- } \\
\text { naik }\end{array}$ & $\begin{array}{c}\text { turun } \\
\text { tetap }\end{array}$ & $\begin{array}{l}\text { tetap- } \\
\text { turun }\end{array}$ & $\begin{array}{l}\text { tetap- } \\
\text { tetap }\end{array}$ \\
\hline & naik-turun & & 2 & & & & & & & \\
\hline & turun-naik & & 1 & 3 & & & & & & \\
\hline & naik-naik & 3 & 1 & 18 & & 1 & & & & \\
\hline PREDIKSI & turun-turun & & & & & & & 1 & & \\
\hline & naik-tetap & & & & & & 2 & & & \\
\hline & tetap-naik & & & & & & & & & \\
\hline & turun-tetap & & & & & & & & & \\
\hline & tetap-turun & & & & & & & & & \\
\hline & tetap-tetap & & & & & & & & & \\
\hline
\end{tabular}

AKTUAL xr (1990-2005)

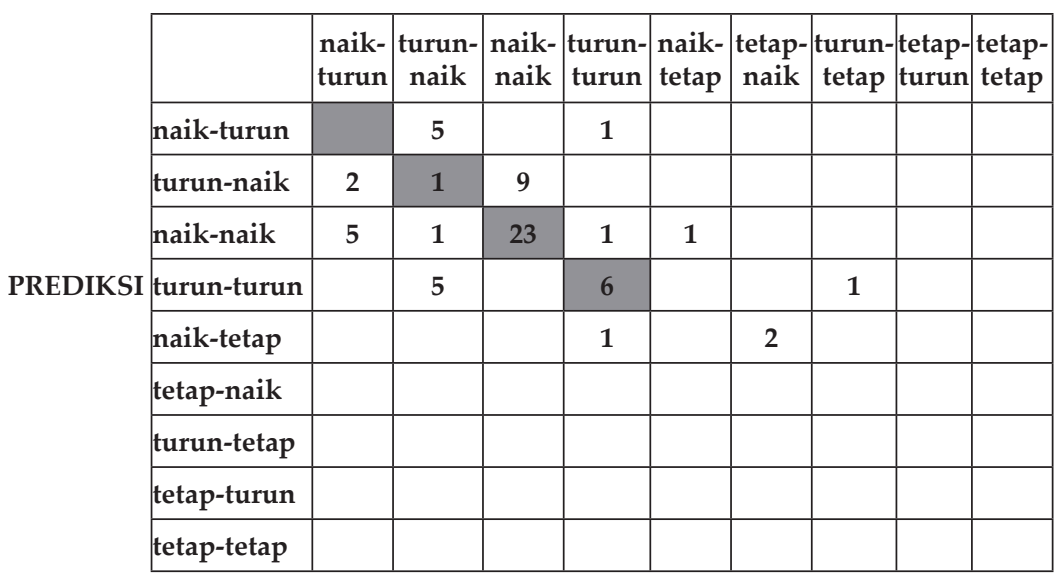

AKTUAL xr (1990-2008)

\begin{tabular}{|l|l|c|c|c|c|c|c|c|c|c|}
\hline & $\begin{array}{c}\text { naik- } \\
\text { turun }\end{array}$ & $\begin{array}{c}\text { turun- } \\
\text { naik }\end{array}$ & $\begin{array}{c}\text { naik- } \\
\text { naik }\end{array}$ & $\begin{array}{c}\text { turun- } \\
\text { turun }\end{array}$ & $\begin{array}{c}\text { naik- } \\
\text { tetap }\end{array}$ & $\begin{array}{c}\text { tetap- } \\
\text { naik }\end{array}$ & $\begin{array}{c}\text { turun- } \\
\text { tetap }\end{array}$ & $\begin{array}{c}\text { tetap- } \\
\text { turun }\end{array}$ & $\begin{array}{c}\text { tetap- } \\
\text { tetap }\end{array}$ \\
\hline naik-turun & & 5 & & 1 & & & & & \\
\hline turun-naik & 3 & 1 & 13 & & & & & & \\
\hline & naik-naik & 8 & 1 & 23 & 1 & 1 & & & & \\
\cline { 2 - 11 } & turun-turun & & 7 & & 7 & & & 1 & & \\
\hline & naik-tetap & & & & 1 & & 2 & & & \\
\hline tetap-naik & & & & & & & & & \\
\hline turun-tetap & & & & & & & & & \\
\hline tetap-turun & & & & & & & & & \\
\hline tetap-tetap & & & & & & & & & \\
\hline
\end{tabular}




\begin{tabular}{|l|c|c|c|c|c|c|c|c|c|}
\hline & $\begin{array}{c}\text { naik- } \\
\text { turun }\end{array}$ & turun- & naik- & turun- & naik- & tetap- & turun- & tetap- & tetap- \\
naik & turun & tetap & naik & tetap & turun & tetap \\
\hline naik-turun & & 2 & & 1 & & & & & \\
\hline turun-naik & 4 & & 2 & & & & & & \\
\hline naik-naik & 5 & & 6 & & & & & & \\
\hline turun-turun & & 6 & & 6 & & & & & \\
\hline naik-tetap & & & & & & & & & \\
\hline tetap-naik & & & & & & & & & \\
\hline turun-tetap & & & & & & & & & \\
\hline tetap-turun & & & & & & & & & \\
\hline tetap-tetap & & & & & & & & & \\
\hline
\end{tabular}

AKTUAL INFLASI (1990-2005)

\begin{tabular}{|l|c|c|c|c|c|c|c|c|c|}
\hline & $\begin{array}{c}\text { naik- } \\
\text { turun }\end{array}$ & $\begin{array}{c}\text { turun- } \\
\text { naik }\end{array}$ & $\begin{array}{c}\text { naik- } \\
\text { naik }\end{array}$ & $\begin{array}{c}\text { turun- } \\
\text { turun }\end{array}$ & $\begin{array}{c}\text { naik- } \\
\text { tetap }\end{array}$ & $\begin{array}{c}\text { tetap- } \\
\text { naik }\end{array}$ & $\begin{array}{c}\text { turun- } \\
\text { tetap }\end{array}$ & $\begin{array}{l}\text { tetap- } \\
\text { turun }\end{array}$ & $\begin{array}{l}\text { tetap- } \\
\text { tetap }\end{array}$ \\
\hline naik-turun & & 4 & & 3 & & & & & \\
\hline turun-naik & 7 & & 8 & & & & & & \\
\hline naik-naik & 8 & & 12 & & & & & & \\
\hline turun-turun & & 8 & & 11 & & & & & \\
\hline naik-tetap & & & & & & & & & \\
\hline tetap-naik & & & 1 & & & & & & \\
\hline turun-tetap & & & & & & 1 & & & \\
\hline tetap-turun & & & & 1 & & & & & \\
\hline tetap-tetap & & & & & & & & & \\
\hline
\end{tabular}

AKTUAL INFLASI (1990-2008)

\begin{tabular}{|l|c|c|c|c|c|c|c|c|c|}
\hline & $\begin{array}{c}\text { naik- } \\
\text { turun }\end{array}$ & $\begin{array}{c}\text { turun- } \\
\text { naik }\end{array}$ & $\begin{array}{c}\text { naik- } \\
\text { naik }\end{array}$ & $\begin{array}{c}\text { turun- } \\
\text { turun }\end{array}$ & $\begin{array}{c}\text { naik- } \\
\text { tetap }\end{array}$ & $\begin{array}{c}\text { tetap- } \\
\text { naik }\end{array}$ & $\begin{array}{c}\text { turun- } \\
\text { tetap }\end{array}$ & $\begin{array}{l}\text { tetap- } \\
\text { turun }\end{array}$ & $\begin{array}{c}\text { tetap- } \\
\text { tetap }\end{array}$ \\
\hline naik-turun & & 5 & & 3 & & & & & \\
\hline turun-naik & 8 & & 8 & & & & & & \\
\hline naik-naik & 8 & & 15 & & & & & & \\
\hline turun-turun & & 9 & & 15 & & & & & \\
\hline naik-tetap & & & & & & & & & \\
\hline tetap-naik & & & 1 & & 1 & & & & \\
\hline turun-tetap & & & & & & 2 & & & \\
\hline tetap-turun & & & & 1 & & & & & \\
\hline tetap-tetap & & & & & & & & & \\
\hline
\end{tabular}




\begin{tabular}{|c|c|c|c|c|c|c|c|c|c|c|}
\hline \multicolumn{3}{|c|}{ Moving Average } & \multicolumn{8}{|c|}{ AKTUAL INFLASI (1990-1997) } \\
\hline \multirow{10}{*}{ PREDIKSI } & & $\begin{array}{l}\text { naik- } \\
\text { turun }\end{array}$ & $\begin{array}{l}\text { turun- } \\
\text { naik }\end{array}$ & $\begin{array}{c}\text { naik- } \\
\text { naik }\end{array}$ & $\begin{array}{l}\text { turun- } \\
\text { turun }\end{array}$ & $\begin{array}{l}\text { naik- } \\
\text { tetap }\end{array}$ & $\begin{array}{c}\text { tetap- } \\
\text { naik }\end{array}$ & $\begin{array}{l}\text { turun- } \\
\text { tetap }\end{array}$ & $\begin{array}{l}\text { tetap- } \\
\text { turun }\end{array}$ & $\begin{array}{l}\text { tetap- } \\
\text { tetap }\end{array}$ \\
\hline & naik-turun & 9 & & & & & & & & \\
\hline & turun-naik & & 8 & & & & & & & \\
\hline & naik-naik & & & 8 & & & & & & \\
\hline & turun-turun & & & & 7 & & & & & \\
\hline & naik-tetap & & & & & & & & & \\
\hline & tetap-naik & & & & & & & & & \\
\hline & turun-tetap & & & & & & & & & \\
\hline & tetap-turun & & & & & & & & & \\
\hline & tetap-tetap & & & & & & & & & \\
\hline
\end{tabular}

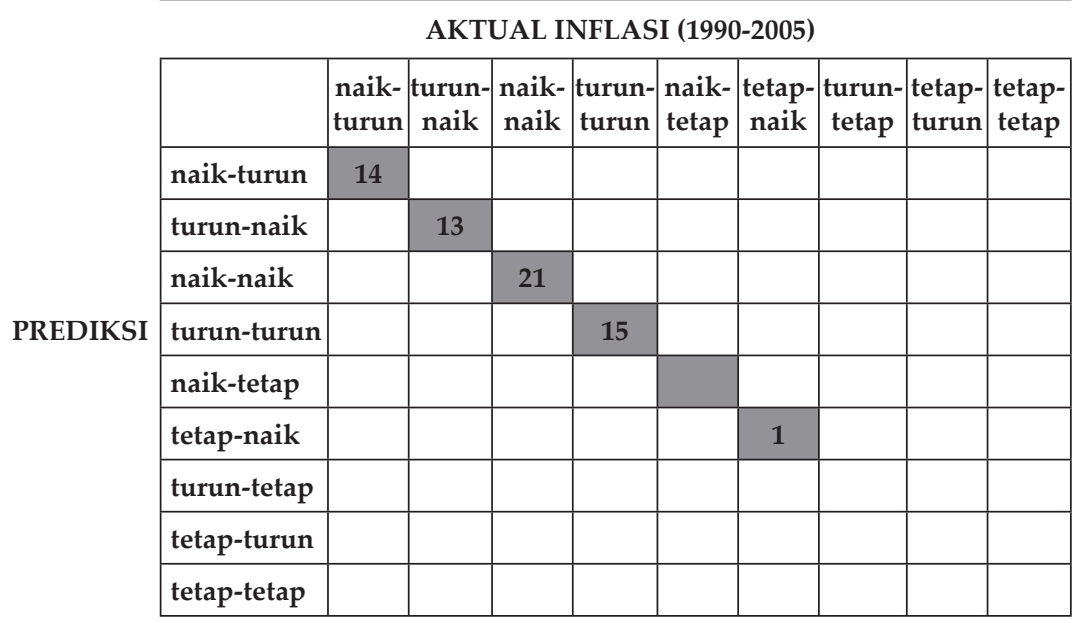

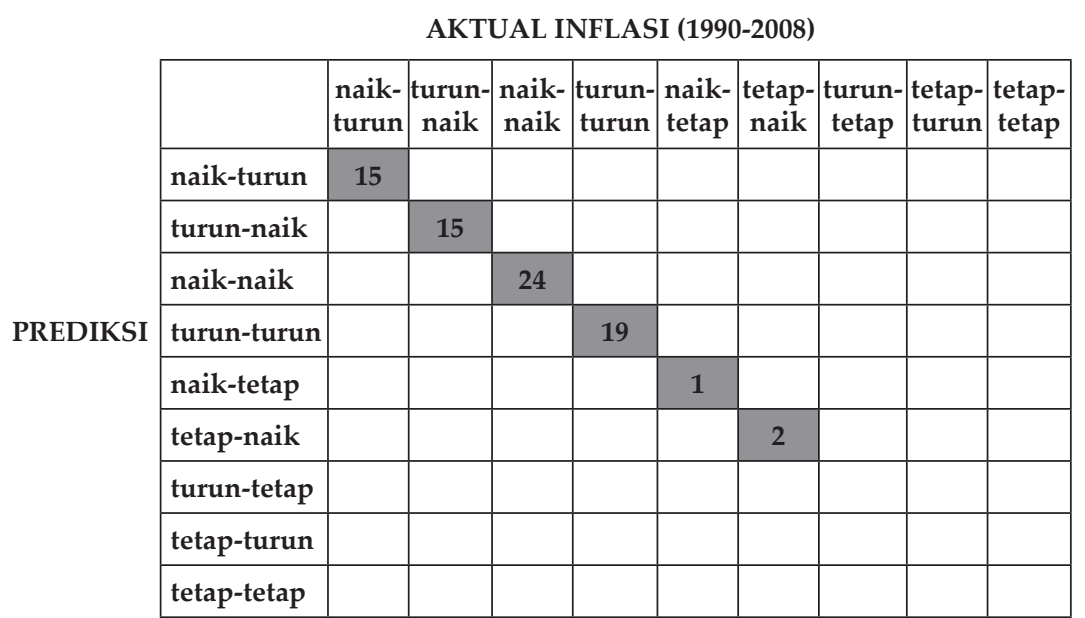


AKTUAL INFLASI (1990-1997)

\begin{tabular}{|l|c|c|c|c|c|c|c|c|c|}
\hline & $\begin{array}{c}\text { naik- } \\
\text { turun }\end{array}$ & $\begin{array}{c}\text { turun- } \\
\text { naik }\end{array}$ & $\begin{array}{c}\text { naik- } \\
\text { naik }\end{array}$ & $\begin{array}{c}\text { turun- } \\
\text { turun }\end{array}$ & $\begin{array}{c}\text { naik- } \\
\text { tetap }\end{array}$ & $\begin{array}{c}\text { tetap- } \\
\text { naik }\end{array}$ & $\begin{array}{c}\text { turun- } \\
\text { tetap }\end{array}$ & $\begin{array}{c}\text { tetap- } \\
\text { turun }\end{array}$ & $\begin{array}{c}\text { tetap- } \\
\text { tetap }\end{array}$ \\
\hline naik-turun & & 1 & & 1 & & & & & \\
\hline turun-naik & 3 & 2 & 1 & & & & & & \\
\hline naik-naik & 4 & & 6 & & & & & & \\
\hline turun-turun & & 6 & & 6 & & & & & \\
\hline naik-tetap & & & & & & & & & \\
\hline tetap-naik & & & & & & & & & \\
\hline turun-tetap & & & & & & & & & \\
\hline tetap-turun & & & & & & & & & \\
\hline tetap-tetap & & & & & & & & & \\
\hline
\end{tabular}

AKTUAL INFLASI (1990-2005)

\begin{tabular}{|l|c|c|c|c|c|c|c|c|c|}
\hline & $\begin{array}{l}\text { naik- } \\
\text { turun }\end{array}$ & $\begin{array}{l}\text { turun- } \\
\text { naik }\end{array}$ & $\begin{array}{l}\text { naik- } \\
\text { naik }\end{array}$ & $\begin{array}{l}\text { turun- } \\
\text { turun }\end{array}$ & $\begin{array}{l}\text { naik- } \\
\text { tetap }\end{array}$ & $\begin{array}{l}\text { tetap- } \\
\text { naik }\end{array}$ & $\begin{array}{l}\text { turun- } \\
\text { tetap }\end{array}$ & $\begin{array}{l}\text { tetap- } \\
\text { turun }\end{array}$ & $\begin{array}{l}\text { tetap } \\
\text { tetain }\end{array}$ \\
\hline naik-turun & & 2 & & 3 & & & & & \\
\hline turun-naik & 6 & 2 & 7 & & & & & & \\
\hline naik-naik & 6 & & 10 & & 1 & & & & \\
\hline turun-turun & & 9 & & 9 & & & & & \\
\hline naik-tetap & & & & & & & & & \\
\hline tetap-naik & & & 1 & & & & & & \\
\hline turun-tetap & & & & & & 1 & & & \\
\hline tetap-turun & & & & 1 & & & & & \\
\hline tetap-tetap & & & & & & & & & \\
\hline
\end{tabular}

AKTUAL INFLASI (1990-2008)

\begin{tabular}{|l|c|c|c|c|c|c|c|c|c|}
\hline & $\begin{array}{c}\text { naik- } \\
\text { turun }\end{array}$ & $\begin{array}{c}\text { turun- } \\
\text { naik }\end{array}$ & $\begin{array}{c}\text { naik- } \\
\text { naik }\end{array}$ & $\begin{array}{c}\text { turun- } \\
\text { turun }\end{array}$ & $\begin{array}{c}\text { naik- } \\
\text { tetap }\end{array}$ & $\begin{array}{c}\text { tetap- } \\
\text { naik }\end{array}$ & $\begin{array}{c}\text { turun- } \\
\text { tetap }\end{array}$ & $\begin{array}{l}\text { tetap- } \\
\text { turun }\end{array}$ & $\begin{array}{l}\text { tetap- } \\
\text { tetap }\end{array}$ \\
\hline naik-turun & & 3 & & 3 & & & & & \\
\hline turun-naik & 7 & 2 & 7 & & & & & & \\
\hline naik-naik & 6 & & 13 & & 1 & & & & \\
\hline turun-turun & & 10 & & 13 & & & & & \\
\hline naik-tetap & & & & & & & & & \\
\hline tetap-naik & & & 1 & & 1 & & & & \\
\hline turun-tetap & & & & & & 2 & & & \\
\hline tetap-turun & & & & 1 & & & & & \\
\hline tetap-tetap & & & & & & & & & \\
\hline
\end{tabular}


Appendix 2: Predicted and Actual Macro Indicators

\section{Indonesian Narrow Money (M1)}
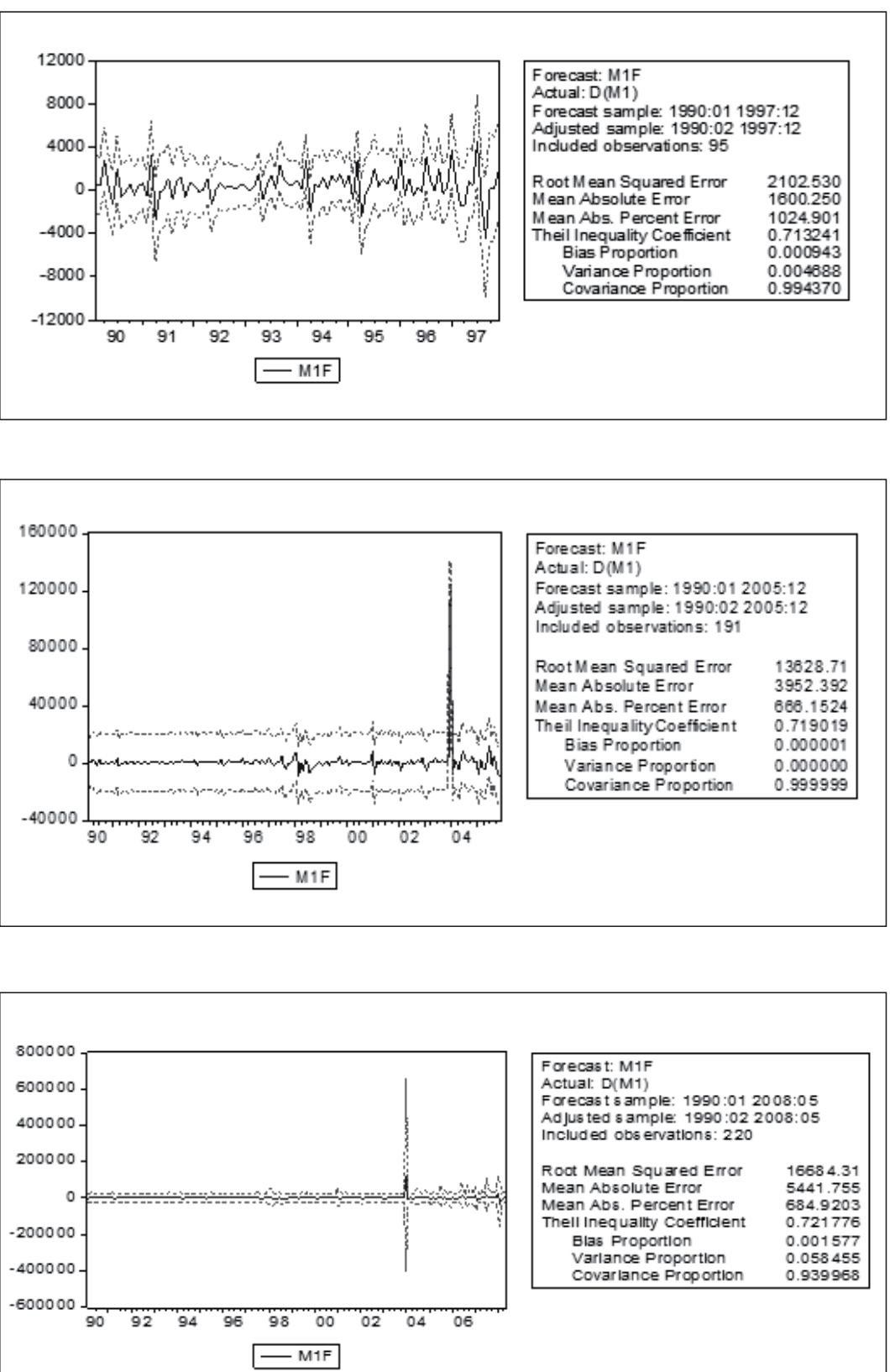

\begin{tabular}{|c|c|}
\hline $\begin{array}{l}\text { Forecast: M1F } \\
\text { Actual: D(M1) } \\
\text { Forecas t } 8 \text { ample: } 1990: 01 \\
\text { Ad Jus ted } 8 \text { ample: } 1990: 02 \\
\text { Included obs ervations: } 220\end{array}$ & $\begin{array}{l}8: 05 \\
8: 05\end{array}$ \\
\hline Root Mean Squared Error & 16684.31 \\
\hline Mean Absolute Error & 5441.755 \\
\hline Mean Abs. Percent Error & 684.9203 \\
\hline Thell Inequallty coefficlent & 0.721776 \\
\hline Blas Proportion & 0.001577 \\
\hline Varlance Proportion & 0.058455 \\
\hline Covarlance Proportion & 0.939968 \\
\hline
\end{tabular}

IJMS 17 (Special Issue), 119-141 (2010) 139 


\section{Bank Indonesia Rate}

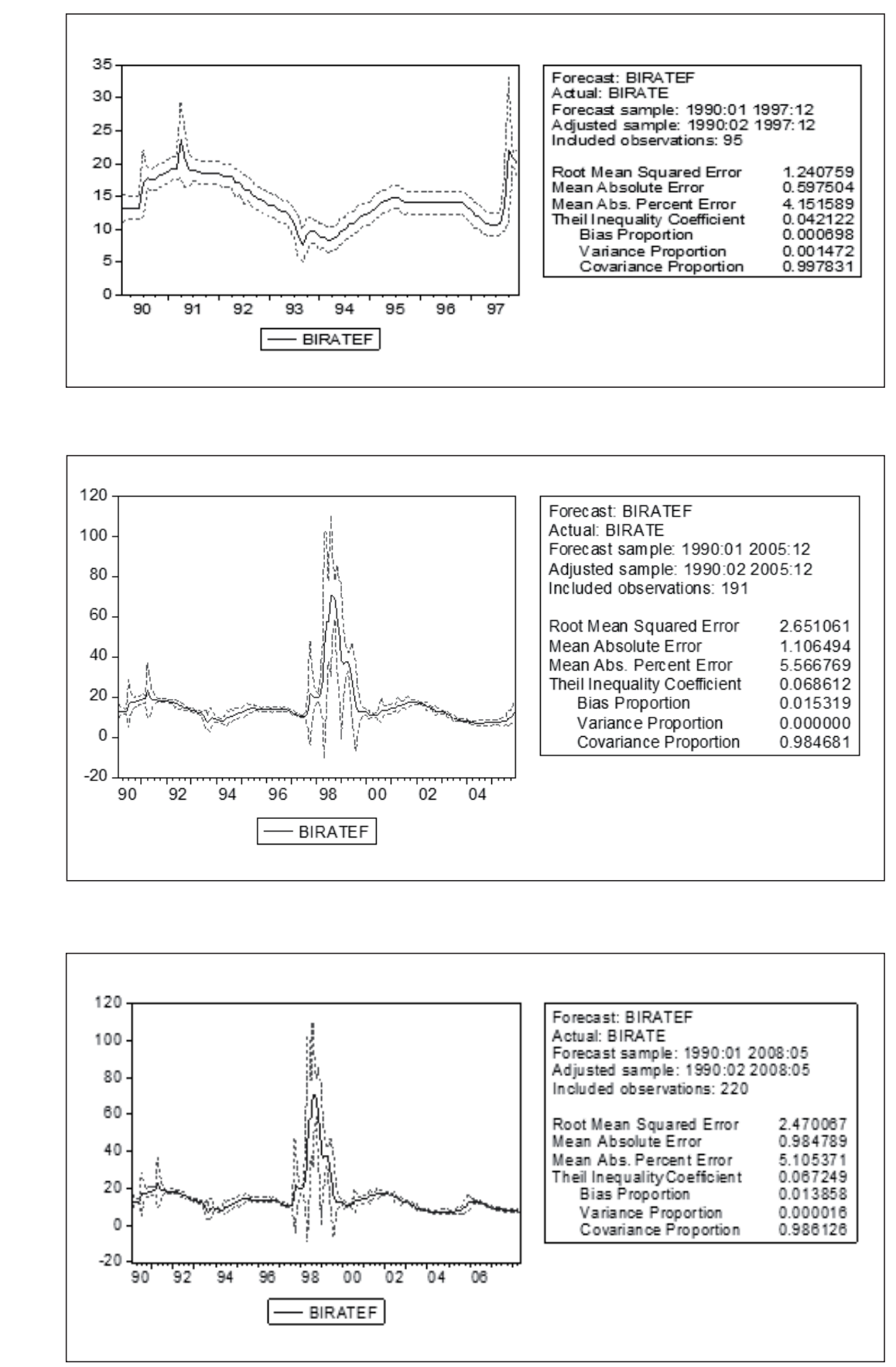

140 IJMS 17 (Special Issue), 119-141 (2010) 


\section{Exchange Rates}
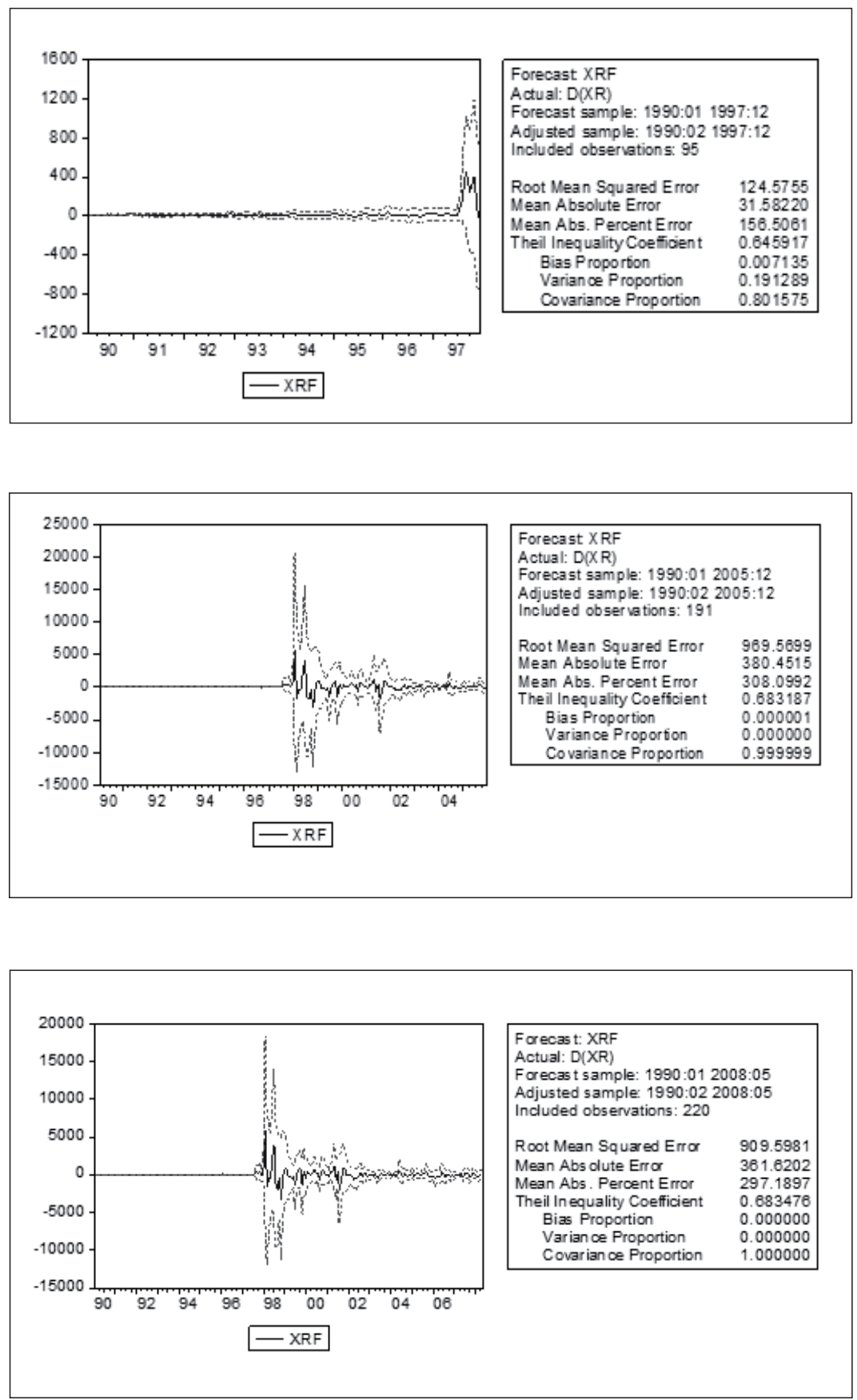

IJMS 17 (Special Issue), 119-141 (2010) 141 